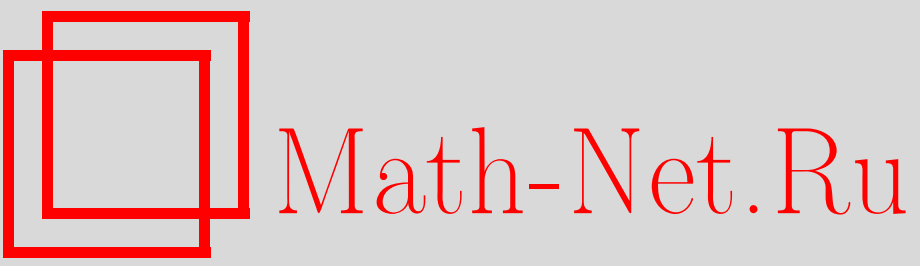

И. П. Денисова, Исследование модели цилиндрического источника гравитационного поля, ТМФ, 1997, том 113, номер 1, 162-167

DOI: https://doi.org/10.4213/tmf1073

Использование Общероссийского математического портала Math-Net.Ru подразумевает, что вы прочитали и согласны с пользовательским соглашением

http://www . mathnet.ru/rus/agreement

Параметры загрузки:

IP: 35.174 .16 .151

26 апреля 2023 г., 12:50:50 


\section{ИССЛЕДОВАНИЕ МОДЕЛИ ЦИЛИНДРИЧЕСКОГО ИСТОЧНИКА ГРАВИТАЦИОННОГО ПОЛЯ}

Построена модель электромагнитного источника для уравнений релятивистской теории гравитации и найдено их точное решение. Проведен анализ асимптотики этого решения.

В работе [1] было найдено точное решение уравнений релятивистской теории гравитации. В цилиндрических координатах интервалы псевдоевклидова пространства-времени $d \sigma^{2}$ и эффективного псевдориманова пространства-времени $d s^{2}$ для этого решения выбирались в виде

$$
\begin{aligned}
d \sigma^{2}= & c^{2} d t^{2}-d r^{2}-r^{2} d \varphi^{2}-d z^{2} \\
d s^{2}= & {[1+W(r, \varphi)] c^{2} d t^{2}+2 s W(r, \varphi) c d t d z-} \\
& -d r^{2}-r^{2} d \varphi^{2}-[1-W(r, \varphi)] d z^{2}
\end{aligned}
$$

где $s= \pm 1$.

Интегрирование уравнений гравитационного поля привело к следующему выражению для $W(r, \varphi)$ вне источника:

$$
W(r, \varphi)=C_{0} K_{0}\left(\frac{m c r}{\hbar}\right)+2 \sum_{n=1}^{\infty} C_{n} K_{n}\left(\frac{m c r}{\hbar}\right) \cos \left(n \varphi+\psi_{n}\right)
$$

где $K_{n}(x)$ - модифицированная функция Бесселя третьего рода, $m$ - масса гравитона, $\hbar$ - постоянная Планка, а $C_{0}, C_{n}$ и $\psi_{n}$ - произвольные постоянные, численные значения которых можно определить, сшивая внешнее решение с внутренним на границе источника.

Из выражений (1) и уравнения связи

$$
\sqrt{-g} g^{i k}=\sqrt{-\gamma}\left[\gamma^{i k}+\Phi^{i k}\right]
$$

следует, что компоненты тензора гравитационного поля $\Phi^{i k}$ явно зависят от функции $W(r, \varphi)$ :

$$
\Phi^{00}=\Phi^{33}=-W(r, \varphi), \quad \Phi^{03}=s W(r, \varphi)
$$

* Московский государственный авиационный технологический университет, Москва, Россия 
Для того чтобы решение (1) удовлетворяло принципу причинности, функция $W(r, \varphi)$ не должна принимать положительных значений: $W(r, \varphi) \leq 0$. Поэтому возникает вопрос, сушествуют ли в природе материальные источники, метрика которых имеет вид (1) с функцией $W(r, \varphi)$, удовлетворяющей условию $W(r, \varphi) \leq 0$ во всех точках пространства.

Построению одной из возможных моделей источника гравитационного поля, удовлетворяющих всем перечисленным требованиям, посвящена настоящая работа.

Для выяснения природы источника гравитационного поля (1) отметим следующее. Постоянные $C_{0}$ и $C_{n}$, входяшие в выражение (2), должны быть непосредственно связаны с распределением материи в источнике, в том числе и с его плотностью энергии. Поэтому исследование законов преобразования коэффициентов $C_{0}$ и $C_{n}$ при преобразованиях Лоренца может дать нам информацию о законах преобразования плотности энергии источника и тем самым о возможной природе источника. Наиболее удобно совершить преобразование Лоренца вдоль оси z с постоянной скоростью $v$ :

$$
t=\frac{t^{\prime}+v z^{\prime} / c^{2}}{\sqrt{1-\frac{v^{2}}{c^{2}}}}, \quad r=r^{\prime}, \quad \varphi=\varphi^{\prime}, \quad z=\frac{z^{\prime}+v t^{\prime}}{\sqrt{1-\frac{v^{2}}{c^{2}}}} .
$$

В результате такого преобразования в штрихованной системе отсчета метрический тензор псевдоевклидова пространства $\gamma_{i k}$ не изменится, а вместо функции $W(r, \varphi)$ в выражение для $d s^{\prime 2}$ будет входить функция

$$
W^{\prime}(r, \varphi)=\frac{(1+s v / c)}{(1-s v / c)}\left[C_{0} K_{0}(m r)+2 \sum_{n=1}^{\infty} C_{n} K_{n}\left(\frac{m c r}{\hbar}\right) \cos \left(n \varphi+\psi_{n}\right)\right] .
$$

Так как $v / c<1$, то, сравнивая выражения (2) и (4), можно заметить, что преобразование $(3)$ привело лишш к появлению множителя $(1+s v / c) /(1-s v / c)$ у констант $C_{0}, C_{n}$ и тем самым изменило их величину (увеличило при $s=+1$ и уменьшило при $s=-1$ ), но не изменило знак самих констант.

Такой закон преобразования констант по своему виду напоминает закон преобразования плотности энергии электромагнитного поля со взаимно перпендикулярными и равными по величине векторами напряженностей $\vec{E}$ и $\vec{H}$ при движении системы отсчета перпендикулярно плоскости, в которой расположены эти векторы.

Поэтому примем в качестве модели источника гравитационного поля (1) электромагнитное поле, векторы напряженности $\vec{E}$ и $\vec{H}$ которого лежат в плоскости $X O Y$ и удовлетворяют условиям: $(\vec{E} \vec{H})=0,|\vec{E}|=|\vec{H}|$. Очевидно, что плотность заряда $\rho$ и вектор плотности тока $\vec{J}$, создающие такое электромагнитное поле, можно представить в виде

$$
\rho=\rho(r, \varphi), \quad \vec{J}=\vec{e}_{z} J(r, \varphi)
$$

Так как $\rho(r, \varphi)$ и $J(r, \varphi)$ не зависят ни от времени, ни от координаты $z$, то дифференциальный закон сохранения заряда выполняется автоматически. Однако функции $\rho(r, \varphi)$ и $J(r, \varphi)$ не могут быть произвольными, а должны обеспечить ортогональность и равенство по абсолютной величине создаваемых ими в псевдоримановом пространстве-времени векторов $\vec{E}$ и $\vec{H}$. 
Для нахождения связи между $\rho(r, \varphi)$ и $J(r, \varphi)$ рассмотрим следуюшую задачу. Предположим, что в некоторой цилиндрической области пространства $r \leq b$ находятся электрические заряды с плотностью $\rho=\rho(r, \varphi)$ и, кроме того, в этой областиплотность электрического тока отлична от нуля и имеет вид $\vec{J}=J(r, \varphi) \vec{e}_{z}$. Выясним условия, при которых точное решение уравнений релятивистской теории гравитации и электродинамики приводит к интервалу вида (1).

Запишем систему уравнений

$$
\begin{gathered}
R_{i k}=\frac{m^{2} c^{2}}{2 \hbar^{2}}\left[g_{i k}-\gamma_{i k}\right]+\frac{2 G}{c^{4}}\left\{-F_{i l} F_{k}^{\cdot l}+\frac{1}{4} g_{i k} F_{l m} F^{l m}\right\}, \\
\frac{\partial F_{i k}}{\partial x^{l}}+\frac{\partial F_{k l}}{\partial x^{i}}+\frac{\partial F_{l i}}{\partial x^{k}}=0, \\
\frac{1}{\sqrt{-g}} \frac{\partial \sqrt{-g} F^{i k}}{\partial x^{k}}=-\frac{4 \pi}{c} J^{i} .
\end{gathered}
$$

Учитывая приведенные вьше соображения, будем считать, что ненулевыми компонентами тензора электромагнитного поля являются

$$
F_{0 r}=E_{r}, \quad F_{0 \varphi}=E_{\varphi}, \quad F_{z r}=-H_{\varphi}, \quad F_{z \varphi}=H_{r},
$$

где $E_{\varphi}$ и $H_{\varphi}$ - геометрические, а не физические компоненты векторов $\vec{E}$ и $\vec{H}$. В этом случае уравнения релятивистской теории гравитации (5) при $i=k=0$ и $i=k=3$ примут вид

$$
\begin{aligned}
R_{00}= & \frac{1}{2}\left\{\frac{\partial^{2} W(r, \varphi)}{\partial r^{2}}+\frac{1}{r} \frac{\partial W(r, \varphi)}{\partial r}+\frac{1}{r^{2}} \frac{\partial^{2} W(r, \varphi)}{\partial \varphi^{2}}\right\}=\frac{m^{2} c^{2}}{2 \hbar^{2}} W(r, \varphi)+ \\
& +\frac{G}{c^{4} r^{2}}\left\{W(r, \varphi)^{2}\left[E_{\varphi}^{2}-2 s E_{\varphi} H_{r}+E_{r}^{2} r^{2}+2 s E_{r} H_{\varphi} r^{2}+H_{\varphi}^{2} r^{2}+H_{r}^{2}\right]+\right. \\
& \left.+2 W(r, \varphi)\left[s E_{r} H_{\varphi} r^{2}-s E_{\varphi} H_{r}+H_{\varphi}^{2} r^{2}+H_{r}^{2}\right]+\left[E_{\varphi}^{2}+E_{r}^{2} r^{2}+H_{\varphi}^{2} r^{2}+H_{r}^{2}\right]\right\} \\
R_{z z}= & \frac{1}{2}\left\{\frac{\partial^{2} W(r, \varphi)}{\partial r^{2}}+\frac{1}{r} \frac{\partial W(r, \varphi)}{\partial r}+\frac{1}{r^{2}} \frac{\partial^{2} W(r, \varphi)}{\partial \varphi^{2}}\right\}=\frac{m^{2} c^{2}}{2 \hbar^{2}} W(r, \varphi)+ \\
& +\frac{G}{c^{4} r^{2}}\left\{W(r, \varphi)^{2}\left[E_{\varphi}^{2}-2 s E_{\varphi} H_{r}+E_{r}^{2} r^{2}+2 s E_{r} H_{\varphi} r^{2}+H_{\varphi}^{2} r^{2}+H_{r}^{2}\right]+\right. \\
& \left.+2 W(r, \varphi)\left[s E_{\varphi} H_{r}-E_{\varphi}^{2}-E_{r}^{2} r^{2}-s E_{r} H_{\varphi} r^{2}\right]+\left[E_{\varphi}^{2}+E_{r}^{2} r^{2}+H_{\varphi}^{2} r^{2}+H_{r}^{2}\right]\right\} .
\end{aligned}
$$

Вычитая из первого уравнения системы (6) второе, получим

$$
r^{2}\left[H_{\varphi}+s E_{r}\right]^{2}+\left[H_{r}-s E_{\varphi}\right]^{2}=0 .
$$

Таким образом, для совместности системы уравнений (6) необходимо потребовать, чтобы выполнялись условия

$$
H_{\varphi}=-s E_{r}, \quad H_{r}=s E_{\varphi} .
$$


Подставляя эти выражения в систему уравнений релятивистской теории гравитации, несложно убедиться, что она сведется к одному уравнению:

$$
\frac{\partial^{2} W}{\partial r^{2}}+\frac{1}{r} \frac{\partial W}{\partial r}+\frac{1}{r^{2}} \frac{\partial^{2} W}{\partial \varphi^{2}}=\frac{m^{2} c^{2}}{\hbar^{2}} W+\frac{4 G}{c^{4} r^{2}}\left\{E_{\varphi}^{2}+E_{r}^{2} r^{2}\right\} .
$$

Обратимся теперь к уравнениям Максвелла (5). Учитьвая соотношения (1) и (7), получим следуюшие уравнения:

$$
\begin{gathered}
\frac{\partial E_{\varphi}}{\partial r}-\frac{\partial E_{r}}{\partial \varphi}=0 \\
\frac{\partial E_{r}}{\partial r}+\frac{E_{r}}{r}+\frac{1}{r^{2}} \frac{\partial E_{\varphi}}{\partial \varphi}=4 \pi \rho(r, \varphi), \\
\frac{\partial E_{r}}{\partial r}+\frac{E_{r}}{r}+\frac{1}{r^{2}} \frac{\partial E_{\varphi}}{\partial \varphi}=-\frac{4 \pi s}{c} J(r, \varphi) .
\end{gathered}
$$

Для совместности этой системы уравнений необходимо и достаточно, чтобы плотность зарядов, находящихся на цилиндрической поверхности, была связана с плотностью тока на этой поверхности соотношением

$$
J(r, \varphi)=-s c \rho(r, \varphi) .
$$

Отсюда видно, что выбор знака $s$ соответствует заданию направления тока при одном и том же знаке $\rho(r, \varphi)$. Кроме того, из выражения (10) следует, что если использовать заряды одного знака для создания требуемой плотности тока, то заряды должны двигаться со скоростью света, что физически невозможно. Поэтому для выполнения условия (10) в цилиндрической области должны находиться заряды обоих знаков, плотности которых $\rho_{p}(r, \varphi)$ и $\rho_{n}(r, \varphi)$ должны быть неравными по абсолютной величине $\left(\rho(r, \varphi)=\left|\rho_{p}(r, \varphi)\right|-\left|\rho_{n}(r, \varphi)\right|\right)$ и векторы скорости которых также должны быть различными. Простейшим примером такой системы является проводяшая поверхность, на которую нанесена некоторая избыточная плотность отрицательных зарядов $\rho(r, \varphi)$ и в создании требуемой плотности тока в которой принимают участие и электроны проводимости.

Для решения поставленной задачи мы должны проинтегрировать уравнение (8) и два первых уравнения системы (9). Вводя потенциалы электромагнитного поля и полагая, как обычно это делается в стационарном случае, что

$$
\vec{E}=-\operatorname{grad} \Phi, \quad \vec{H}=\operatorname{rot} \vec{A},
$$

несложно убедиться, что первое уравнение системы (9) удовлетворяется тождественно, а второе уравнение дает

$$
\frac{\partial^{2} \Phi}{\partial r^{2}}+\frac{1}{r} \frac{\partial \Phi}{\partial r}+\frac{1}{r^{2}} \frac{\partial^{2} \Phi}{\partial \varphi^{2}}=-4 \pi \rho(r, \varphi)
$$


Таким образом, для решения поставленной задачи мы получили два линейных дифференциальных уравнения (8) и (11). Поэтому по заданному распределению зарядов $\rho(r, \varphi)$ и токов $J(r, \varphi)$, удовлетворяющих условию $(10)$, всегда может быть найдено создаваемое ими электромагнитное поле, после чего из уравнения (8) несложно найти функцию $W(r, \varphi)$, которая непосредственно связана с тензором гравитационного поля $\Phi^{i k}$.

Для дальнейшего анализа рассмотрим простейший случай, когда плотности зарядов $\rho(r, \varphi)$ и токов $J(r, \varphi)$ не зависят от полярного угла $\varphi$ и сосредоточены на цилиндрической поверхности радиуса $r=b$.

В этом случае для определения поля удобнее воспользоваться теоремой Гаусса, а не решать уравнение (11).

Обозначая заряд единицы длины цилиндрической поверхности через $\chi$, будем иметь $E_{\varphi}=E_{r}=0$ при $r<b$ и $E_{\varphi}=0, E_{r}=2 \chi / r$ при $r \geq b$. Подставляя эти выражения в уравнение (8), получим однородное уравнение при $r<b$ и неоднородное уравнение

при $r \geq b$.

$$
\frac{\partial^{2} W}{\partial r^{2}}+\frac{1}{r} \frac{\partial W}{\partial r}-\frac{m^{2} c^{2}}{\hbar^{2}} W=\frac{16 G \chi^{2}}{c^{4} r^{2}}
$$

Линейно независимыми решениями однородного уравнения (12), как известно, являются $K_{0}(m c r / \hbar)$ и $I_{0}(m c r / \hbar)$. Вронскиан функций $K_{0}(x)$ и $I_{0}(x)$ равен

$$
K_{0}^{\prime}(x) I_{0}(x)-I_{0}^{\prime}(x) K_{0}(x)=-\frac{1}{x} .
$$

Поэтому решение неоднородного уравнения (12) мы можем записать в виде

$$
W=-K_{0}\left(\frac{m c r}{\hbar}\right) \int_{c_{2}}^{r} r d r f(r) I_{0}\left(\frac{m c r}{\hbar}\right)+I_{0}\left(\frac{m c r}{\hbar}\right) \int_{c_{1}}^{r} r d r f(r) K_{0}\left(\frac{m c r}{\hbar}\right),
$$

где $f(r)$ - правая часть уравнения (12), а $c_{1}$ и $c_{2}$ - произвольные постоянные. Так как функция $f(r)$ всюду конечна, то естественно потребовать, чтобы и решение уравнения было несингулярным. Это можно сделать, выбирая соответствующим образом постоянные $c_{1}$ и $c_{2}$. Для этого учтем, что при $x \rightarrow 0$ функция $I_{0}(x)$ стремится к единице, а функция $K_{0}(x)$ неограниченно возрастает. При $x \rightarrow \infty$ уже функция $I_{0}(x)$ неограниченно возрастает, в то время как $K_{0}(x)$ стремится к нулю. Несложно убедиться, что для получения несингулярного решения уравнения (12) необходимо выбрать $c_{1}=\infty$, $c_{2}=0$. Поэтому для $W(r)$ будем иметь

$$
W=-K_{0}\left(\frac{m c r}{\hbar}\right) \int_{0}^{r} r d r f(r) I_{0}\left(\frac{m c r}{\hbar}\right)-I_{0}\left(\frac{m c r}{\hbar}\right) \int_{r}^{\infty} r d r f(r) K_{0}\left(\frac{m c r}{\hbar}\right) .
$$

Так как в рассматриваемом нами случае $f(r)=16 G \chi^{2} /\left(c^{4} r^{2}\right)$ при $r \geq b$ и $f(r)=0$ при $r<b$, то отсюда получим непрерывное решение:

$$
\begin{aligned}
W= & -\frac{16 G \chi^{2}}{c^{4}} I_{0}\left(\frac{m c r}{\hbar}\right) \int_{b}^{\infty} \frac{d r}{r} K_{0}\left(\frac{m c r}{\hbar}\right) \quad \text { при } r<b, \\
W= & -\frac{16 G \chi^{2}}{c^{4}}\left\{K_{0}\left(\frac{m c r}{\hbar}\right) \int_{b}^{r} \frac{d r}{r} I_{0}\left(\frac{m c r}{\hbar}\right)+\right. \\
& \left.+I_{0}\left(\frac{m c r}{\hbar}\right) \int_{r}^{\infty} \frac{d r}{r} K_{0}\left(\frac{m c r}{\hbar}\right)\right\} \quad \text { при } r \geq b .
\end{aligned}
$$


Прямой подстановкой можно убедиться, что дополнительные уравнения (см. [2]) релятивистской теории гравитации $D_{i}\left[\sqrt{-g} g^{i k}\right]=0$ для полученного нами решения (13) выполняются тождественно.

Следует отметить, что при $b \rightarrow 0$ выражения (13) теряют смысл, так как входящие в них интегралы расходятся. Это связано с тем, что в этом случае плотность энергии электромагнитного поля, являющаяся источником для функции $W(r)$, обрашается в бесконечность на оси цилиндра пропорционально $r^{-2}$ и эта особенность является неинтегрируемой. Таким образом, обращение функции $W(r)$ в бесконечность при $b \rightarrow 0$ есть следствие некорректной с математической точки зрения постановки задачи.

Используя асимптотические выражения функций $I_{0}(x)=\exp (x) / \sqrt{2 \pi x}$ и $K_{0}(x)=$ $\sqrt{\pi / 2 x} \exp (-x)$, справедливые при $x \rightarrow \infty$, из второго соотношения (13) найдем, что при $r \gg \hbar / m c$ функция $W(r)$ имеет асимптотику:

$$
W(r)=-\frac{16 G \chi^{2} \hbar^{2}}{m^{2} c^{6} r^{2}} .
$$

Так как в области $0 \leq x<\infty$ функции $K_{0}(x)$ и $I_{0}(x)$ являются неотрицательными, то из выражений (13) следует, что $W(r) \leq 0$ в соответствии с требованиями принципа причинности.

Таким образом, мы показали, что существует большой класс материальных источников, для которых система уравнений Максвелла и релятивистской теории гравитации приводит к метрике вида (1), удовлетворяющей принципу причинности.

\section{Список литературы}

[1] И. П. Денисова. ТМФ. 1997. Т. 112. № 3. С. 501-512.

[2] А. А. Логунов, М.А. Мествиришвили. Основы релятивистской теории гравитации. М.: Наука, 1989.

Поступила в редакцию 24.III.1997 г.

\section{P. Denissova \\ INVESTIGATION OF THE CYLINDRICAL SOURCE MODEL OF THE GRAVITATIONAL FIELD}

The model of the electromagnetic source for equations of the relativistic theory of gravitation is constructed and their exact solution is found. An analysis of the asymptotic behaviour of the solution is considered. 